\title{
Interplay of Optical Force and Ray-Optic Behavior between Luneburg Lenses
}

\author{
Alireza Akbarzadeh, ${ }^{* \dagger}$ J. A. Crosse, ${ }^{\ddagger}$ Mohammad Danesh, ${ }^{\ddagger}$, Cheng-Wei Qiu, ${ }^{\ddagger}$ Aaron J. Danner, \\ and Costas M. Soukoulis ${ }^{\dagger}, \|$ \\ ${ }^{\dagger}$ Institute of Electronic Structure and Laser, Foundation for Research and Technology-Hellas, Heraklion, Crete, Greece 71110 \\ ${ }^{\ddagger}$ Department of Electrical and Computer Engineering, National University of Singapore, 4 Engineering Drive 3, Singapore 117576 \\ ${ }^{\S}$ Electronics and Photonics Department, Institute of High Performance Computing, 1 Fusionopolis Way, Singapore 138632 \\ "Ames Laboratory and Department of Physics and Astronomy, Iowa State University, Ames, Iowa 50011, United States
}

ABSTRACT: The method of force tracing is employed to examine the optomechanical interaction between two and four Luneburg lenses. Using a simplified analytical model, as well as a realistic numerical model, the dynamics of elastic and fully inelastic collisions between the lenses under the illumination of collimated beams are studied. It is shown that elastic collisions cause a pair of Luneburg lenses to exhibit oscillatory and translational motion simultaneously. The combination of these two forms of motion can be used to optomechanically manipulate small particles. Additionally, it is addressed how fully inelastic collisions of four Luneburg lenses can help us achieve full transparency as well as isolating space to trap particles.
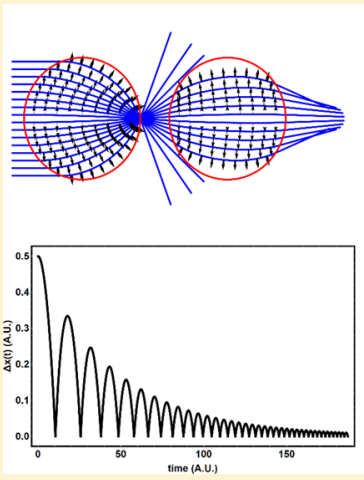
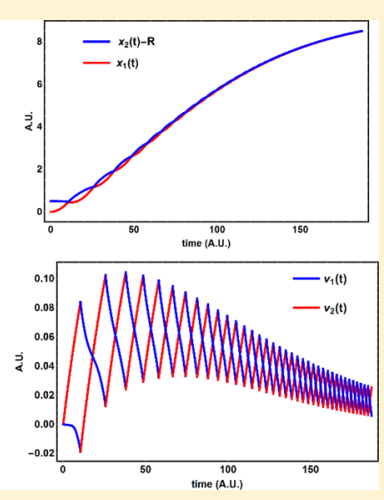

KEYWORDS: optical force, geometrical optics, optical manipulation, graded-index media, metamaterials

$\mathrm{T}$ he mechanical properties of light have been the topic of debate from the early stages of the emergence of electromagnetic wave theory. ${ }^{1-5}$ The optomechanical properties of light have been the subject of much study, but despite this, the area of optical force is still an active area of research, and specifically, the enigma of the momentum of light in a medium is still under scrutiny. ${ }^{6-11}$ The well-known AbrahamMinkowski dilemma, the definition of the Poynting vector, and the interpretation of the stress tensor as well as its relation to the optical force and torque densities are the major concerns of the current fundamental studies on the optical force. In spite of these theoretical uncertainties, optical forces have been employed practically in fascinating applications: microscopy and optical imaging, ${ }^{12,13}$ optical tweezers and particle trapping, ${ }^{14-16}$ optical tractor beams and optical lift, ${ }^{17-22}$ light driven motors, ${ }^{23}$ and radiation pressure on optical cloaks ${ }^{24,25}$ are a few examples out of many. With the advent of new fabricating and characterization technology and development of theoretical studies, it is expected that optical force will play a big role in novel applications in the near future.

The conventional approach to calculate the optical force in a medium is to solve the Maxwell equations for the electromagnetic fields, construct the elements of the stress tensor, and integrate the divergence of the stress tensor over the volume of the interest. ${ }^{26,27}$ However, applying this standard full-wave method in complex media can be technically hard or intensively time-consuming. Alternatively, by taking advantage of the ease provided by the geometrical optics and relying on its sufficient accuracy under some restrictions, which are met in many scenarios, the authors proposed a new method called "force tracing" to trace the optical force field along the trajectories of light in a complex medium. ${ }^{28}$ In ref 28 , the authors took the electromagnetic fields as quasi-plane waves with slowly changing amplitudes and rapidly fluctuating phases as

$$
\left\{\begin{array}{l}
\vec{E}(\vec{r}, t)=\vec{E}_{0} \exp \left(i k_{0} \vec{k} \cdot \vec{r}-i \omega t\right) \\
\vec{H}(\vec{r}, t)=\vec{H}_{0} \exp \left(i k_{0} \vec{k} \cdot \vec{r}-i \omega t\right)
\end{array}\right.
$$

where $\omega$ is the angular frequency, $\vec{k}$ is the wave vector, $k_{0}=\omega / c$, $c$ is the speed of light in free space, and both $\vec{E}_{0}$ and $\vec{H}_{0}$ are vectors with approximately constant magnitudes. On the basis of this assumption, which is the core of geometrical optics, and with the help of the Hamiltonian-based ray equations, ${ }^{29,30}$ after a lengthy algebraic manipulation, the Lorentz force density in a lossless isotropic medium is simplified to

$$
\langle\vec{f}\rangle_{\text {normalized }}=\frac{1}{n^{4}} \vec{k} \times \vec{L}_{\mathrm{k}}
$$

where $n$ is the refractive index and $\vec{L}_{\mathrm{k}}=\hat{e}_{z}\left[k_{x}\left(\mathrm{~d} k_{y} / \mathrm{d} \tau\right)-k_{y}\right.$ $\left.\left(\mathrm{d} k_{x} / \mathrm{d} \tau\right)\right]$. It should be mentioned that the force density in eq 2 has been normalized by $\varepsilon_{0}\left|\vec{E}_{0}\right|^{2} / 2$, with $\varepsilon_{0}$ being the free space

Received: June 26, 2015

Published: August 24, 2015 
(a)

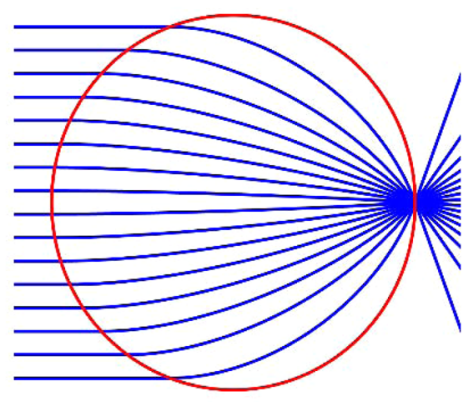

(b)

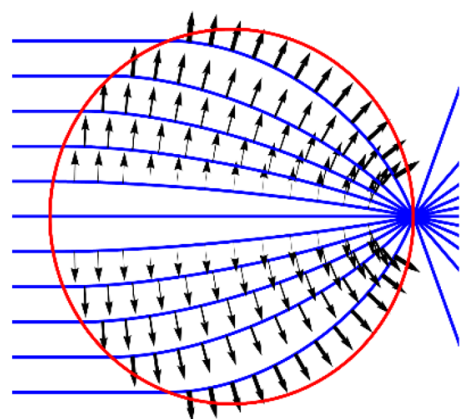

(c)

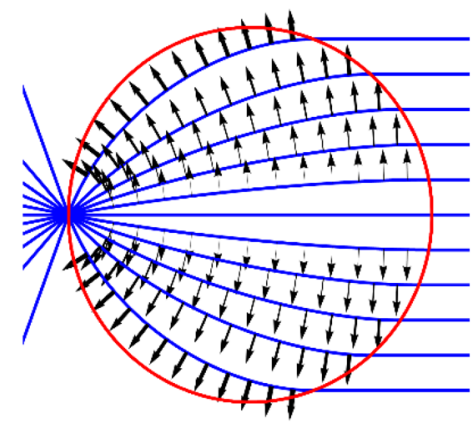

(d)

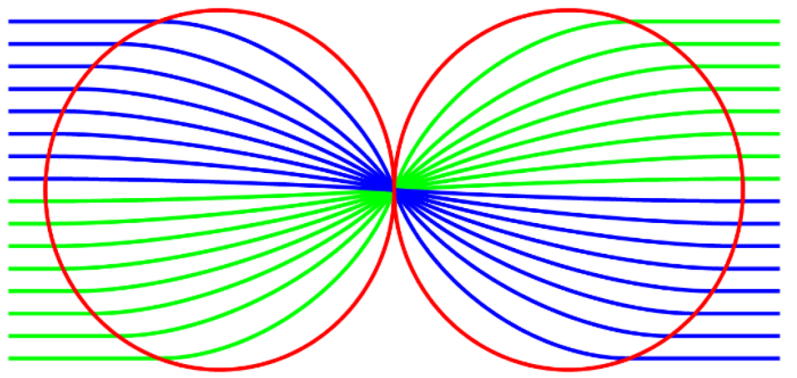

(e)

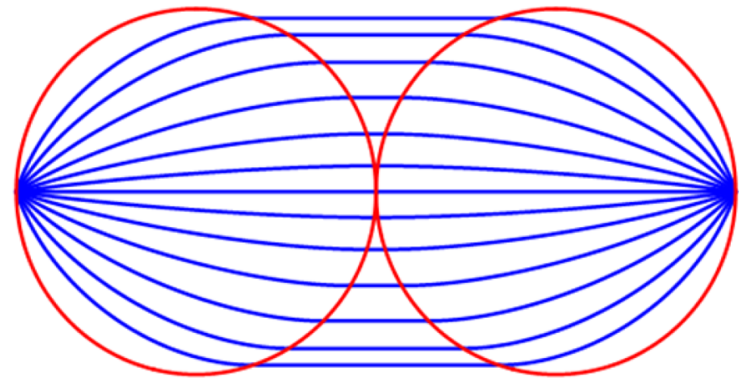

Figure 1. (a) Ray trajectories in a Luneburg lens; (b) a collimated beam shining from left-side pushes the Luneburg lens; (c) rays entering from a single point pull the Luneburg lens; (d) the performance of two touching Luneburg lenses; (e) the complementarity of two touching Luneburg lenses. A point located on one of the two Luneburg lenses is imaged perfectly on the other one.

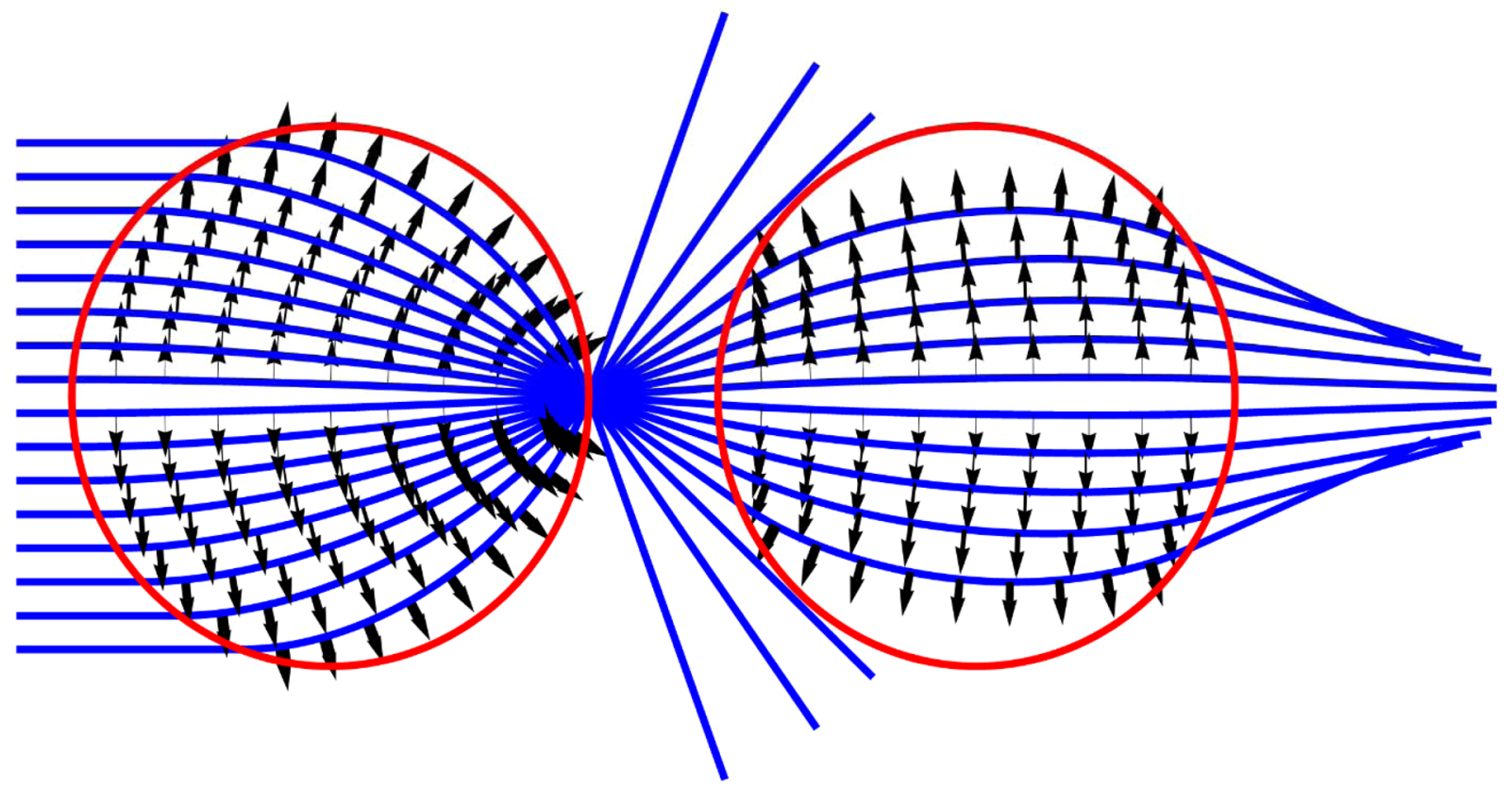

Figure 2. Optical force field applied by the light rays illuminating from left side onto two nontouching Luneburg lenses. The optical force on the first Luneburg lens is always pushing and constant. The optical force on the second lens can be pushing, pulling, or null, subject to its distance from the first lens.

permittivity. An interested reader is referred to ref 28 for more details on the derivation of eq 2 , as well as the proposed formulation for the force tracing in anisotropic media and the surface force density at the interfaces between two different media. However, it should be noted that the anisotropic formulation in ref 28 is true only for the cases with diagonal constitutive tensors, and for nondiagonal cases, it should be modified.

In this article, we use force tracing technique to study the dynamics of optomechanical interaction between Luneburg 
lenses under illumination of collimated light beams. As will be discussed later, owing to the spatial variation of optical forces acting on a system made up of more than one Luneburg lens, the collision between the lenses causes oscillatory and translational motions. Both elastic and fully inelastic collisions will be considered, and through a simplified analytical as well as a more realistic numerical model, the equation of motion will be derived and discussed in detail. The collision of Luneburg lenses under illumination of light leads to optical transparency, space isolation, and spatially temporally modulated light beams. These results are interesting for a wide range of researchers and can have important applications in biosensing, particle imaging, particle transport, and particle trapping.

\section{DISCUSSION AND RESULTS}

A Luneburg lens ${ }^{29}$ is a spherically symmetric graded-index lens with radius $R_{0}$ and profile index $n(r)=\left(2-\left(r / R_{0}\right)^{2}\right)^{1 / 2}$, which

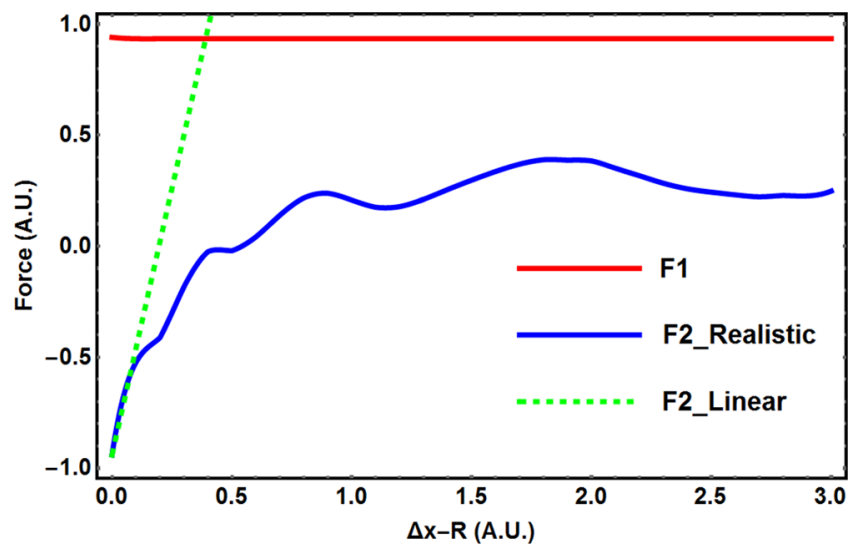

Figure 3. Total optical force applied on the two Luneburg lenses calculated via the full-wave simulation and the approximated linear optical force acting on the second lens for $\alpha=4.65 \times 10^{-12}$. It is assumed that the summation of the Luneburg lenses' radii is equal to $R$. When $\Delta x-R=0$, the lenses are touching.

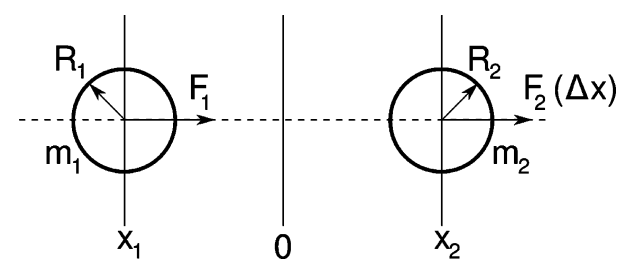

Figure 4. Schematic of the two Luneburg lenses that are colliding due to a collimated light beam illuminated from left side.

focuses parallel rays shining from one side to a single point (Figure 1a). Using the force tracing technique, it can be seen that the parallel rays entering the lens would exert a positive force ( $a$ force in the direction of the light ray) on it (Figure 1b). However, as shown in Figure 1c, if rays enter into the lens from a single point, they actually apply a negative force onto it, and this is due to the fact that the horizontal components of the momentum of the rays gradually increase after departing the lens. If we have an even number of Luneburg lenses touching each other sequentially, the total change in the momentum of light entering parallel to a line cutting the touching points would be zero and the combination of Luneburg lenses do not feel any force. In other words, from the geometrical optics point of view two Luneburg lenses are complementary media (a)

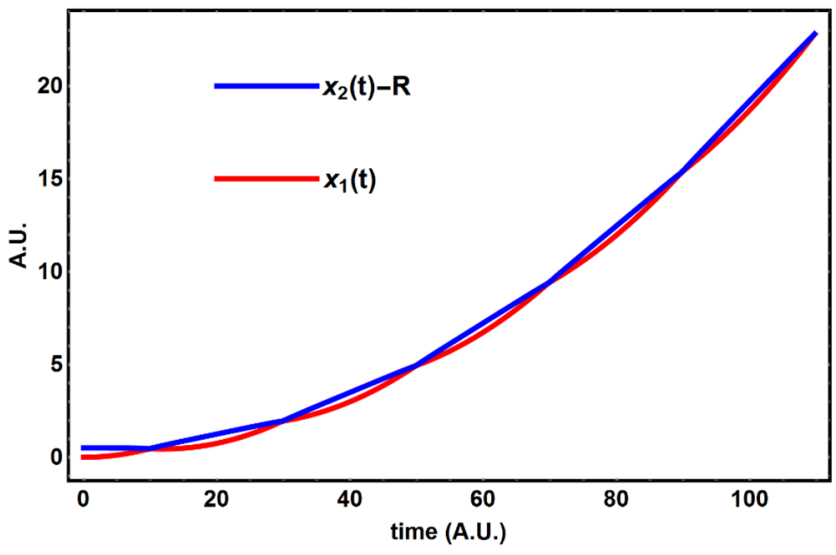

(b)

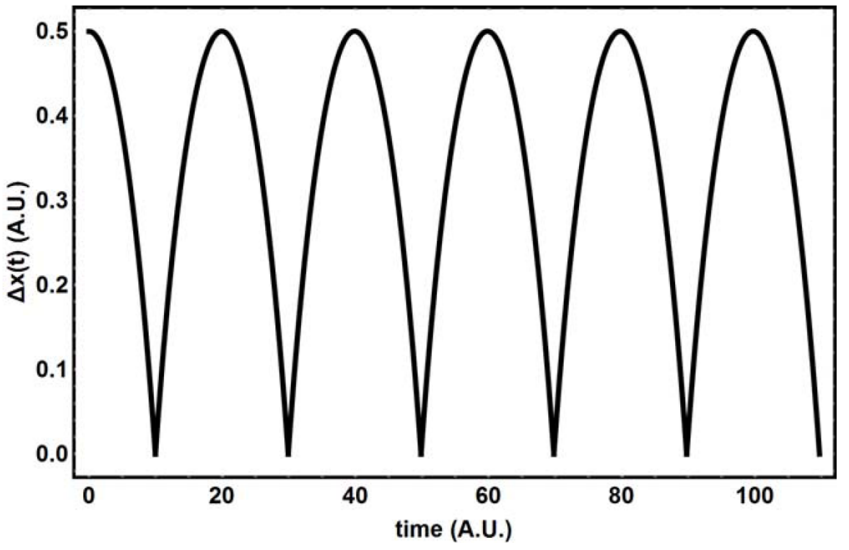

Figure 5. (a) Paths of the two spheres obtained from the realistic numerical model for six collisions. At the collision moments, the two curves touch each other. (b) The corresponding distance between the two spheres versus time for the six collisions $\left(m=10^{-10}, R_{1}=R_{2}=1\right.$, $x_{1,0}=0$, and $x_{2,0}-R=0.5$ ).

and a point source on one of the lenses is perfectly imaged on the other lens (Figure 1e). As an example (see Figure 1d), if we have two Luneburg lenses, after traveling through this combination, the exiting rays look as if there existed no object in front of them (aside from $180^{\circ}$ rotation), and hence, we achieve transparency with inversion. However, if the lenses detach from each other, not all the rays that impinge on the first lens would reach the second one, and consequently, full transparency would be absent. In addition, the momentum of light would alter during its journey through the separated lenses and Luneburg lenses have some optically mediated interaction with each other.

By studying the force on each lens, as shown in Figure 2, we see that as all the incoming rays arrive at the first lens, the force on the first lens is always constant and acts in the direction of the wave vector of light. However, the optical force on the second lens is a function of the distance from the first one and can be attractive, repulsive, or vanishing. Figure 3 shows the total force acting on each lens as a function of the separation, as calculated via a full-wave simulation in COMSOL. It can be seen that the force on the second lens is attractive for small separations, and hence, the second lens can become trapped close to the first lens. 
(a)

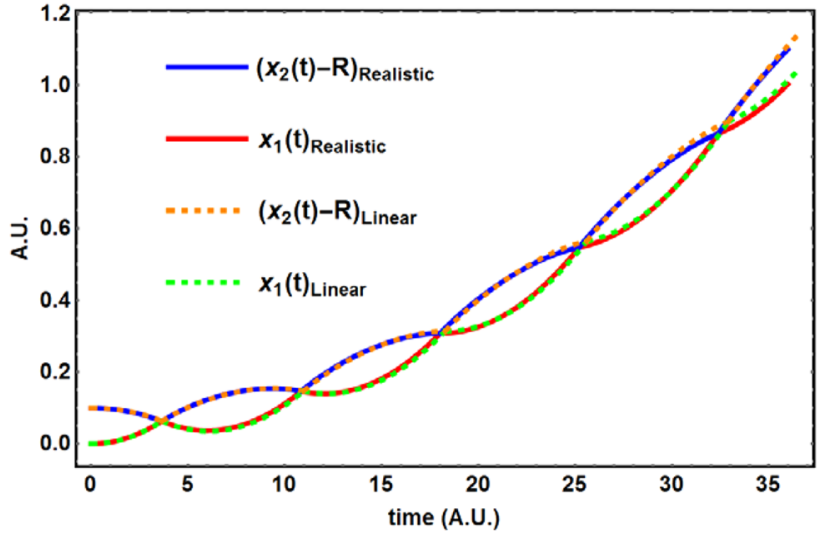

(b)

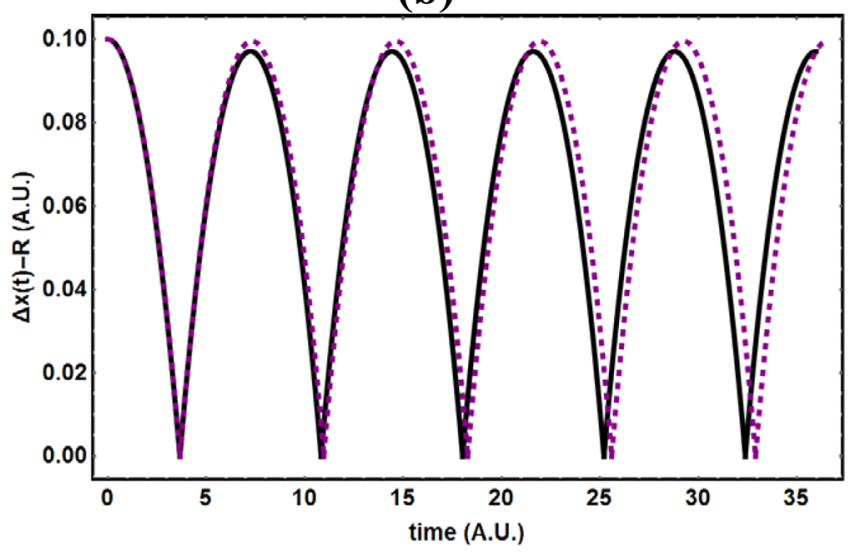

Figure 6. (a) Paths of the two spheres obtained from the simplified analytical model and the realistic model for five collisions. At the collision moments, the two curves touch each other. (b) The corresponding distance between the two spheres vs time for the five collisions, where the solid-line graph is representing the realistic model and the dashed-line curve representing the analytical model $(m=$ $10^{-10}, R_{1}=R_{2}=1, F_{1}=9.4 \times 10^{-13}, x_{1,0}=0, x_{2,0}-R=0.1$, and $\alpha=$ $\left.4.65 \times 10^{-12}\right)$

As seen in Figure 3, the force acting on the second lens is a complicated function of position. In order to obtain a quantitative understanding of the motion of the lenses, let us assume that the force on the first lens is constant, $F_{1}\left(x_{1}\right)=F$, and the force on the second lens is a linear function of the separation, $F_{2}=-F+\alpha(\Delta x-R)$, where the separation is given by $\Delta x=x_{2}-x_{1}$, with $R=R_{1}+R_{2}$ the minimum separation owing to the finite radius of the lenses. Here, $\alpha$ is a scaling factor and should be chosen with care in order to make the linear model resemble a realistic scenario. This form of the force is analytically tractable but preserves the basic phenomenology of the system and is a reasonable approximation for small separations where the force on the second lens remains negative. The approximated linear force for $\alpha=$ $4.65 \times 10^{-12}$ is shown in Figure 3 as well. In the following we consider two lenses of identical mass $m_{1}=m_{2}=m$ and radius $R_{1}$ $=R_{2}=R / 2$, with initial positions and velocities, $x_{1,0}, x_{2,0}$ and $u_{1,0}$ and $u_{2,0}$ for the two lenses, respectively.

The constant, positive force $F_{1}$ causes the first lens to undergo linear motion. However, the force acting on the second lens $F_{2}$ is a negative restoring force that leads to the collision between the two lenses and, hence, an oscillatory (a)

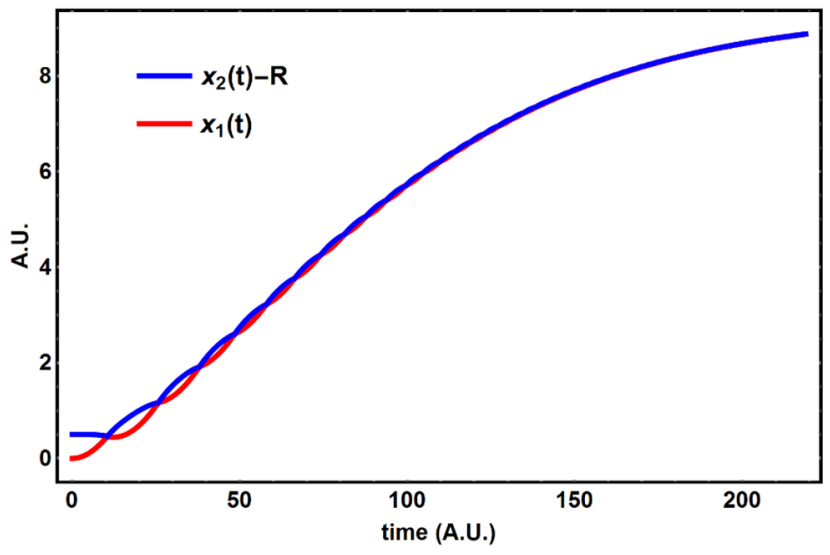

(b)

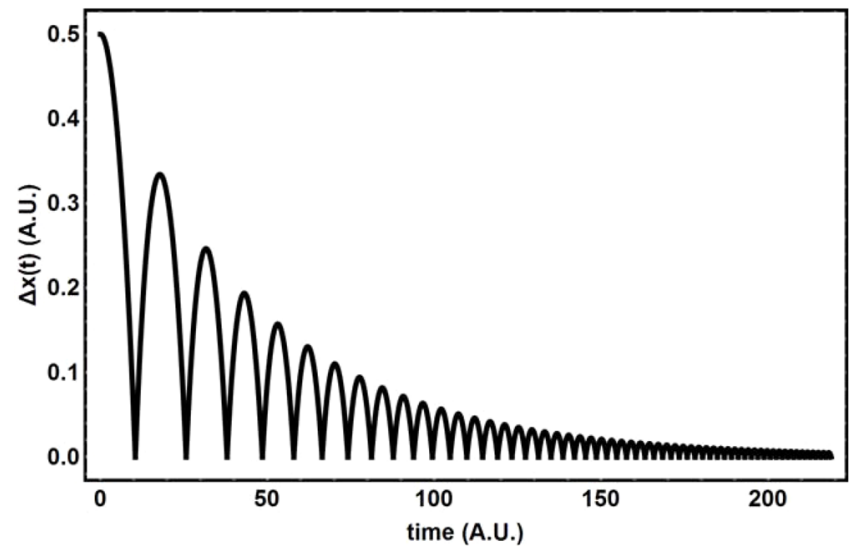

Figure 7. (a) Paths of the two spheres obtained from the realistic numerical model under the influence of damping. (b) The corresponding distance between the two spheres vs time ( $m=$ $10^{-10}, R_{1}=R_{2}=1, x_{1,0}=0, x_{2,0}-R=0.5$, and $\left.k_{\mathrm{d}}=0.03\right)$.

motion. It proves to be convenient to change variables from the absolute coordinates of the spheres to the center-of-mass coordinates and the separation. In this coordinate system, the linear and oscillatory motions separate and, hence, can be solved individually.

The equation of motion for the separation $\Delta x$ reads (see Figure 4)

$$
\frac{\mathrm{d}^{2} \Delta x}{\mathrm{~d} t^{2}}=\frac{\mathrm{d}^{2} x_{2}}{\mathrm{~d} t^{2}}-\frac{\mathrm{d}^{2} x_{1}}{\mathrm{~d} t^{2}}=\frac{F_{2}-F_{1}}{m}=\frac{\alpha(\Delta x-R)-2 F}{m}
$$

which solves to

$$
\begin{aligned}
\Delta v & =\frac{\mathrm{d} \Delta x}{\mathrm{~d} t}=\frac{\alpha\left(\Delta x_{0}-R\right)-2 F}{\sqrt{\alpha m}} \sinh \left[\sqrt{\frac{\alpha}{m}} t\right] \\
& +\Delta u_{0} \cosh \left[\sqrt{\frac{\alpha}{m}} t\right]
\end{aligned}
$$

and

$$
\begin{aligned}
\Delta x & =\frac{1}{\alpha}\left[\alpha\left(\Delta x_{0}-R\right)-2 F\right] \cosh \left[\sqrt{\frac{\alpha}{m}} t\right]+\Delta u_{0} \sqrt{\frac{m}{\alpha}} \sinh \left[\sqrt{\frac{\alpha}{m}} t\right] \\
& +\frac{2 F}{\alpha}+R
\end{aligned}
$$


(a)

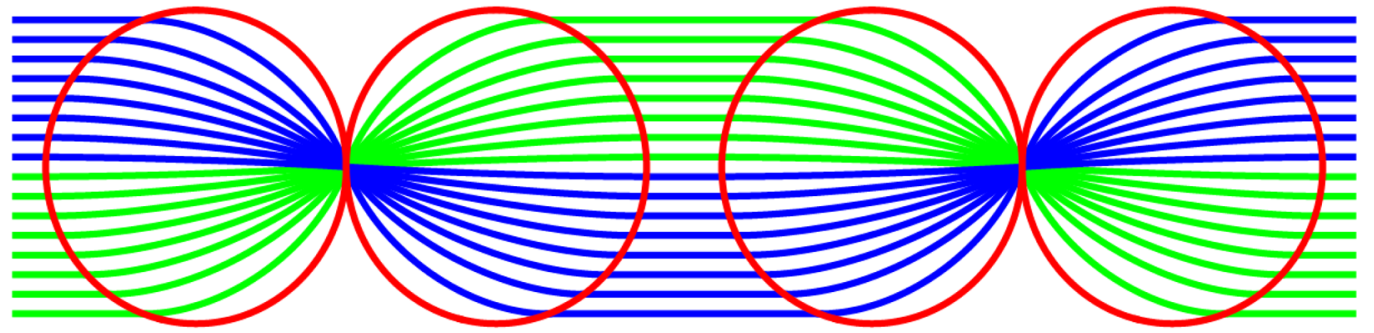

(b)

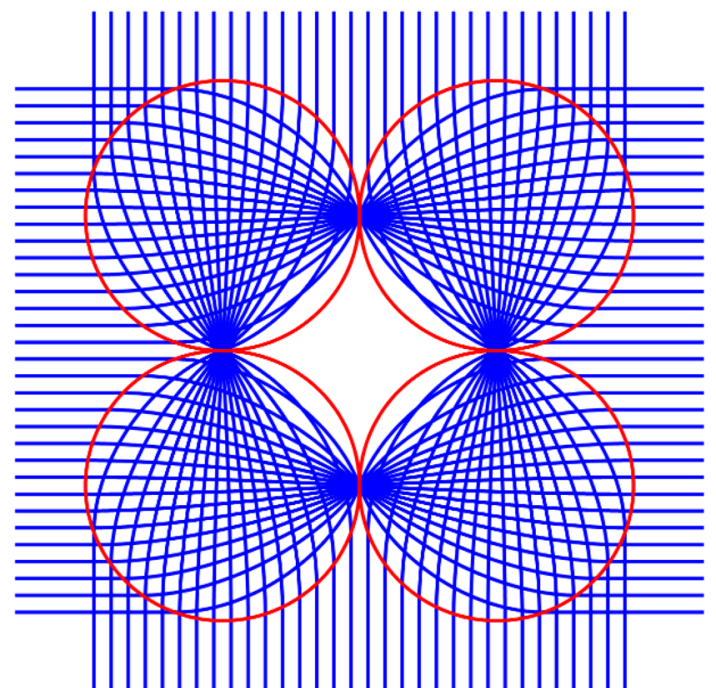

Figure 8. (a) Full transparency achieved by inelastic collision of two pairs of Luneburg lenses; (b) inelastic collision of four Luneburg lenses under vertical and horizontal shining of the collimated light beams to make an isolated area in between.

where $\Delta x_{0}=x_{2,0}-x_{1,0}$ and $\Delta u_{0}=u_{1,0}-u_{2,0}$ are the initial separation and initial approach velocity, respectively.

Let us consider the case where the lenses start at rest $u_{1,0}=$ $u_{2,0}=0$ (hence, $\Delta u_{0}=0$ ). The first collision occurs when the separation $\Delta x=R$, that is, when

$$
\frac{1}{\alpha}\left[\alpha\left(\Delta x_{0}-R\right)-2 F\right] \cosh \left[\sqrt{\frac{\alpha}{m}} t\right]+\frac{2 F}{\alpha}=0
$$

This leads to a first collision time of

$$
t_{1}=\sqrt{\frac{m}{\alpha}} \operatorname{arccosh}\left[B_{0}\right]
$$

where

$$
B_{0}=\frac{2 F}{2 F-\alpha\left(\Delta x_{0}-R\right)}
$$

In an elastic collision, both the energy and momentum must be conserved. From momentum conservation we have

$$
u_{1,1}+u_{2,1}=v_{1,0}+v_{2,0}
$$

and from energy conservation

$$
u_{1,1}^{2}+u_{2,1}^{2}=v_{1,0}^{2}+v_{2,0}^{2}
$$

which leads to

$$
\begin{aligned}
& u_{1,1}=v_{2,0} \\
& u_{2,1}=v_{1,0}
\end{aligned}
$$

where $v_{1,0}$ and $v_{2,0}$ are the incoming velocities of the lenses before the collision and $u_{1,1}$ and $u_{2,1}$ are the outgoing velocities after the collision. Thus, the outgoing velocity from the first collision is

$$
\Delta u_{1}=-\Delta v_{0}=\frac{2 F}{B_{0} \sqrt{\alpha m}} \sqrt{B_{0}^{2}-1}
$$

The outgoing velocities $\Delta u_{0} \rightarrow \Delta u_{1}=-\Delta v_{0}$, and new initial position $\Delta x_{0} \rightarrow \Delta x_{1}=R$ gives the initial conditions for the second collision.

All the subsequent collisions can be computed recursively. The ith collision occurs at

$$
1-\cosh \left[\sqrt{\frac{\alpha}{m}} t_{i}\right]+\frac{\sqrt{\alpha m}}{2 F} \Delta u_{i-1} \sinh \left[\sqrt{\frac{\alpha}{m}} t_{i}\right]=0
$$

which gives a collision time of

$$
t_{i}=\sqrt{\frac{m}{\alpha}} \operatorname{arccosh}\left[B_{i-1}\right]+t_{i-1}
$$

where 


$$
B_{i-1}=\frac{4 F^{2}+\alpha m \Delta u_{i-1}^{2}}{4 F^{2}-\alpha m \Delta u_{i-1}^{2}} \quad \text { for } \quad i>2
$$

and a separation velocity of

$$
\Delta u_{i}=\frac{2 F}{\sqrt{\alpha m}} \sqrt{B_{i-1}^{2}-1}-B_{i-1} \Delta u_{i-1} \quad \text { for } \quad i>2
$$

As can be seen from this analytical formulation, the two lenses, in the absence of damping, undergo an infinite number of elastic collisions as long as the light is shining. However, if the effect of damping is present, which is the case in realistic situations, the model needs to be modified. In order to take the damping into account, we introduce a damping factor $k_{\mathrm{d}}$ in the differential eq 3 ,

$$
\frac{\mathrm{d}^{2} \Delta x}{\mathrm{~d} t^{2}}+k_{\mathrm{d}} \frac{\mathrm{d} \Delta x}{\mathrm{~d} t}=\frac{F_{2}-F_{1}}{m}=\frac{\alpha(\Delta x-R)-2 F}{m}
$$

eq 18 solves to

$$
\Delta x=\left[D_{1} \cosh (\beta t)+D_{2} \sinh (\beta t)\right] e^{-k_{\mathrm{d}} t / 2}+\frac{2 F}{\alpha}+R
$$

where $\beta=\left(4 \alpha+k_{\mathrm{d}}^{2} m / 4 m\right)^{1 / 2}, D_{1}=\left[\alpha\left(\Delta x_{0}-R\right)-2 F\right] / \alpha$, and $D_{2}=\left(2 \Delta u_{0}+k_{\mathrm{d}} D_{1}\right) / 2 \beta$. From eq 19 it is seen that, under the influence of damping, the oscillatory motion of the system decays. Consequently, it is predicted that, as the collisions go on, the range of fluctuations become smaller and smaller; ultimately, the lenses touch each other and the negative restoring force between the lenses vanishes. The oscillations vanish and lenses remain in contact.

The center-of-mass, $x_{\mathcal{c}}$, can be rewritten as $x_{c}=\left(x_{2}+x_{1}\right) / 2=$ $x_{1}+\Delta x / 2$. As the first lens experiences a constant force, the equation of motion for $x_{1}$ is easily solved. Hence, the center-ofmass motion can be written as

$$
\begin{aligned}
& x_{\mathrm{c}}(t)=\frac{F}{2 m} t^{2}+\sum_{i}\left(u_{1, i-1} t+x_{1, i-1}+\Delta x_{i}(t) / 2\right) \\
& \quad \times\left[\chi\left(t-t_{i-1}\right)-\chi\left(t-t_{i}\right)\right]
\end{aligned}
$$

where $\chi(t)$ is the Heaviside step function, that is, $\chi(t)=1$ for $t$ $\geq 0$ and $\chi(t)=0$ for $t<0$, and $\Delta x_{i}$ is computed via the recursive formula above. Thus, we see that the center-of-mass undergoes parabolic acceleration with a correction owing to the interaction between the lenses. As matter of fact, the whole system under the illumination of light exhibits two types of motion: the local oscillatory motion of the two lenses and the global translational motion of the whole system.

However, in the realistic scenario the force applied on the second lens is a complicated function of position. As an analytical solution for this complicated force is not possible, we invoked a numerical analysis to obtain the equation of motion of the two lenses. The result of such analysis is shown in Figure 5a,b. In Figure 5a, where the positions of the two lenses with respect to time for six collisions are presented, the translational and oscillatory motion of the lenses can be clearly observed, a fact expected from the simplified analytical formulation. Shown in Figure $5 b$ is the distance between the spheres versus time, which is a cyclical-shaped curve confirming the oscillatory motion of the spheres. Note that the force acting on the first lens is constant and it may seem that its motion should be a parabolic function with respect to time. However, the velocity of the first lens changes abruptly at the moments of collision, which inflicts discrete corrections on the parabolic equation.
Hence, as seen in Figure 5a, the path of the first lens $x_{1}(t)$ looks like an oscillatory function superimposed on a parabola. The approximated linear model for small initial separations should produce results that are reasonably close to those of the realistic model. For the purpose of accuracy validation, for the case $\Delta x_{0}$ $=0.1$, our results corresponding to the realistic and analytical (with the scaling factor $\alpha=4.65 \times 10^{-12}$ ) models are presented in Figure 6a,b. Note that the linearized $F_{2}(x)$ for $\alpha=4.65 \times$ $10^{-12}$ is shown in Figure 3, where it is seen that the linear and realistic graphs for $F_{2}(x)$ match each other quite well for small separations. Comparing the graphs provided in Figure 6, we see that the analytical model is in good agreement with the numerical model, which certifies the validity of the employed approximation. We believe that the combination of the oscillatory motion and the translational motion of the two lenses can be utilized in applications such as imaging, trapping, or time-space-modulated transportation of small particles. The addition of damping and loss causes the amplitude of the oscillations decay along the translational motion. This is shown in Figure $7 \mathrm{a}, \mathrm{b}$, where the path of the lenses and distance between them are depicted versus time. It is seen in Figure 7 that the period of collisions and the velocity of the lenses decrease as time passes and the lenses approach each other more and more. Eventually the oscillations are damped away and the net force on the whole system of lenses tends to zero, which makes the damping effect dominate and both the translational and oscillatory motions stop.

The dynamic analysis presented above is for the completely elastic collision between the Luneburg lenses. However, if the lenses with velocities $v_{1}$ and $v_{2}$ collide in a completely inelastic way, then after the collision, the lenses carry on their movement together at velocity $\left(v_{1}+v_{2}\right) / 2$. This inelastic collision in principle can be attained by employing mechanisms that cause the lenses to stick together after the first collision, imposing gradually increasing friction on the system or introducing a proper potential barrier. The immediate consequence of such an inelastic collision is the full transparency that we can achieve by combining two pairs of Luneburg lenses, as depicted in Figure 8a. As seen in Figure 8a, due to the complementarity in each pair of Luneburg lenses, the well collimated rays enter the lenses with no reflection, gradually travel through lenses, and exit as if there were no object in front of them. Shown in Figure $8 \mathrm{~b}$ is another setup that can be acquired by the inelastic collision of Luneburg lenses. As noticed in Figure 8b, four Luneburg lenses can be attached together by illuminating collimated beams in horizontal and vertical directions. In this way, the shared space between the lenses is almost empty of light rays and, due to the symmetrical structure of the setup and light rays, the optical force acting on particles within this space is very small or vanishing. This space can be used to trap, sense, or transport particles.

In conclusion, we reviewed the method of force tracing briefly and being inspired by this method we considered the optical force applied on a system consisting an even number of Luneburg lenses. We studied the dynamics of the collision occurring between the lenses under the illumination of collimated light beams analytically and numerically. We showed that for the elastic collision the system exhibits translational and oscillatory motion, while for the fully inelastic case it has only translational motion. Finally, we discussed how effectively such a light-matter interaction can be used in various applications. 


\section{AUTHOR INFORMATION}

\section{Corresponding Author}

*E-mail: alireza.akbarzadeh@iesl.forth.gr.

\section{Notes}

The authors declare no competing financial interest.

\section{ACKNOWLEDGMENTS}

A.A. and C.-W.Q. gratefully appreciate the initial fruitful discussions with Professor Juan José Sáenz. Work at FORTH was supported by the European Research Council under the ERC Advanced Grant No. 320081 (PHOTOMETA). Work at Ames Laboratory was partially supported by the U.S. Department of Energy (Basic Energy Science, Division of Materials Science and Engineering) under Contract No. DEAC02-07CH11358.

\section{REFERENCES}

(1) Maxwell, J. C. A Treatise on Electricity and Magnetism; Clarendon: Oxford, 1904.

(2) Bartoli, A. Il calorico raggiante e il secondo principio di termodinamica. Il Nouvo Cimento 1884, 15, 193-202.

(3) Lebedev, P. N. Investigations on the pressure forces of light. Ann. Phys. 1901, 6, 433-458.

(4) Nichols, E. F.; Hull, G. F. The pressure due to radiation. (Second paper). Phys. Rev. 1903, 17, 26-50.

(5) Nichols, E. F.; Hull, G. F. The pressure due to radiation. Astrophys. J. 1903, 57, 315-351.

(6) Brevik, I. Experiments in phenomenological electrodynamics and the electromagnetic energy-momentum tensor. Phys. Rep. 1979, 52, 133-201.

(7) Leonhardt, U. Optics: Momentum in an uncertain light. Nature (London, U. K.) 2006, 444, 823-824.

(8) Pfeifer, R. N. C.; Nieminen, T. A.; Heckenberg, N. R.; Rubinsztein-Dunlop, H. Colloquium: momentum of an electromagnetic wave in dielectric media. Rev. Mod. Phys. 2007, 79, 11971216.

(9) Baxter, C.; Loudon, R. Radiation pressure and the photon momentum in dielectrics. J. Mod. Opt. 2010, 57, 830-842.

(10) Milonni, P. W.; Boyd, R. W. Momentum of light in a dielectric medium. Adv. Opt. Photonics 2010, 2, 519-553.

(11) Kemp, B. A. Resolution of the Abraham-Minkowski debate: implications for the electromagnetic wave theory of light in matter. J. Appl. Phys. 2011, 109, 111101.

(12) Svoboda, K.; Block, S. M. Biological applications of optical forces. Annu. Rev. Biophys. Biomol. Struct. 1994, 23, 247-285.

(13) Neuman, K. C.; Nagy, A. Single-molecule force spectroscopy: optical tweezers, magnetic tweezers and atomic force microscopy. Nat. Methods 2008, 5, 491-505.

(14) Grier, D. G. A revolution in optical manipulation. Nature (London, U. K.) 2003, 424, 810-816.

(15) Ashkin, A. Acceleration and trapping of particles by radiation pressure. Phys. Rev. Lett. 1970, 24, 156.

(16) Ashkin, A.; Dziedzic, J. M.; Bjorkholm, J. E.; Chu, S. Observation of a single-beam gradient force optical trap for dielectric particles. Opt. Lett. 1986, 11, 288-290.

(17) Chen, J.; Ng, J.; Lin, Z.; Chan, C. T. Optical pulling force. Nat. Photonics 2011, 5, 531-534.

(18) Novitsky, A.; Qiu, C. W.; Wang, H. Single gradientless light beam drags particles as tractor beams. Phys. Rev. Lett. 2011, 107, 203601.

(19) Ruffner, D. B.; Grier, D. G. Optical conveyors: a class of active tractor beams. Phys. Rev. Lett. 2012, 109, 163903.

(20) Brzobohatý, O.; Karásek, V.; Šiler, M.; Chvátal, L.; Čižmár, T.; Zemánek, P. Experimental demonstration of optical transport, sorting and self-arrangement using a 'tractor beam'. Nat. Photonics 2013, 7, 123-127.
(21) Kajorndejnukul, V.; Ding, W.; Sukhov, S.; Qiu, C. W.; Dogariu, A. Linear momentum increases and negative optical forces at dielectric interface. Nat. Photonics 2013, 7, 787-790.

(22) Swartzlander, G. A.; Peterson, T. J.; Artusio-Glimpse, A. B.; Raisanen, A. D. Stable optical lift. Nat. Photonics 2011, 5, 48-51.

(23) Liu, M.; Zentgraf, T.; Liu, Y.; Bartal, G.; Zhang, X. Light-driven nanoscale plasmonic motors. Nat. Nanotechnol. 2010, 5, 570-573.

(24) Chen, H.; Zhang, B.; Luo, Y.; Kemp, B. A.; Zhang, J.; Ran, L.; $\mathrm{Wu}, \mathrm{B}$. I. Lorentz force and radiation pressure on a spherical cloak. Phys. Rev. A: At., Mol., Opt. Phys. 2009, 80, 011808.

(25) Chen, H.; Zhang, B.; Kemp, B. A.; Wu, B. I. Optical force on a cylindrical cloak under arbitrary wave illumination. Opt. Lett. 2010, 35 , 667-669.

(26) Jackson, J. D. Classical Electrodynamics; John Wiley: New York, 1998.

(27) Kong, J. A. Electromagnetic Wave Theory; Wiley: New York, 1986.

(28) Akbarzadeh, A.; Danesh, M.; Qiu, C. W.; Danner, A. J. Tracing optical force fields within graded-index media. New J. Phys. 2014, 16, 053035 .

(29) Leonhardt, U.; Philbin, T. G. Geometry and Light: The Science of Invisibility; Dover Publications: New York, 2010.

(30) Akbarzadeh, A.; Danner, A. J. Generalization of ray tracing in a linear inhomogeneous anisotropic medium: a coordinate-free approach. J. Opt. Soc. Am. A 2010, 27, 2558-2562. 